\title{
Impact of different sensory stimuli on presence in credible virtual environments
}

\author{
Guilherme Gonçalves, Miguel Melo, José Vasconcelos-Raposo and Maximino Bessa
}

\begin{abstract}
Multiple factors can affect presence in virtual environments, such as the number of human senses engaged in a given experience or the extent to which the virtual experience is credible. The purpose of the present work is to study how the inclusion of credible multisensory stimuli affects the sense of presence, namely, through the use of wind, passive haptics, vibration, and scent. Our sample consisted of 37 participants ( 27 men and 10 women) whose ages ranged from 17 to 44 years old and were mostly students. The participants were divided randomly into 3 groups: Control Scenario (visual and auditory $-N=12$ ), Passive Haptic Scenario (visual, auditory, and passive haptic $-\mathrm{N}=13$ ) and Multisensory Scenario (visual, auditory, wind, passive haptic, vibration, and scent $-\mathrm{N}=12$ ). The results indicated a significant increase in the involvement subscale when all multisensory stimuli were delivered. We found a trend where the use of passive haptics by itself has a positive impact on presence, which should be the subject of further work.
\end{abstract}

Index Terms—Virtual Reality, Multisensory, Presence, Immersion, Games, Passive Haptics.

\section{INTRODUCTION}

$\mathrm{V}$ IRTUAL REALITY (VR) is evolving at a quick pace and being adopted successfully in areas such as education [1], [2], [3], training [4], [5], [6] or entertainment [7], [8]. VR can transport its users to a virtual world where they can interact and perceive the experience as real [9]. This feeling of "being there" in the virtual environment is usually referred to in the literature as Presence [9], [10], [11], [12], [13], [14], [15]. Presence is a widely used and accepted metric for evaluating VEs [11], [12], [15], [16], [17], [18].

Moving forward towards multisensory virtual reality (VR) applications is crucial to achieving highly credible environments, which could result in higher levels of presence. The motivation of this work arises from the fact that VR applications fundamentally rely on visual and auditory stimuli and that previous studies conducted regarding multisensory stimuli were conducted in non-credible environments. There is a gap to fill regarding how multisensory stimuli plays their part in creating environment credibility regarding presence. The literature has already shown that multisensory VR applications influence the sense of presence [10], [19], [20], [21], [22]. However, it is important to highlight that merely adding stimulus to a VR application is not enough. If the stimulus that is added is not coherent with the virtual experience, it will result in an extra cognitive load to users and spoil the whole experience [17], [23].

Credibility, as defined by Merriam-Webster [24] "is the quality of inspiring belief". In the present paper, we assume the theoretical proposition that high levels of presence are only possible through the subject's attribution of credibility. To make the most out of VR applications, one must have credible virtual environments (VEs) that are capable of transporting users to a virtual world and having them perceive it as if it is real. Slater and Usoh [16] argue that

- Guilherme Gonçalves, Miguel Melo, José Vasconcelos-Raposo and Maximino Bessa were with the Inesctec Massive Laboratory, University of Trás-os-Montes e Alto Douro, Vila Real.

E-mail: guilhermeg@utad.pt the credibility of the virtual environment can be increased if the virtual world accurately represents the laws of physics. In fact, some studies argue that credibility may increase the levels of presence, leading users to adopt behaviours similar to those displayed when facing real world situations [11], [17], [25], [26]. However, if the virtual world is not credible, the use of coherent sensory input might not be enough to make the experience itself credible [27]. Thus, there is the need to test the multisensory stimuli in environments that by themselves are already credible. Works have already been conducted proving that multisensory stimuli can increase presence [20], [21]. However, the environments used were significantly less immersive in terms of visual graphics and interaction and did not use a variety of stimuli to the same extent as our work. Thus, in the light of recent technologies, we expect that the use of a high number of multisensory stimuli delivered coherently in a credible environment will further increase the sense of presence, like what results suggest in previous works using less-credible environments. We also hypothesise that passive haptics has more impact in developing higher levels of presence when one experiences immersive environments with realistic locomotion provided by today's equipment. We foresee that if participants feel the virtual environment as credible, and can explore it using the same physical movements that they would in reality, they will be more prone to expect certain objects also to be real. Therefore if we also provide passive haptics, their sense of presence should rise.

Our goal is to investigate how multisensory stimulation affects the sense of presence in a credible VE, namely: the combination of visual, audio, wind, passive haptics, vibration, and scent delivered simultaneously, and also the influence of passive haptics alone.

In this work, we propose two hypotheses.

- H1: Adding coherent multisensory stimuli to credible environments will increase the sense of presence.

- H2: The use of passive haptics in credible environments will increase the sense of presence 
To test our hypothesis, we developed a credible VR game. The game allowed the delivery of different multisensory stimuli in a coherent and immersive way. The levels were designed so that we could test the multisensory stimuli of wind, vibration, passive haptic feedback and scent. Moreover, the game levels ensured that players always had a chance to feel the stimuli by directing users to areas where they could feel those stimuli.

This study will contribute to the understanding of how having coherent multisensory stimuli influences presence in credible environments using commercially available offthe-shelf equipment. We also aim to generate knowledge on how passive haptics influences presence in a VE. Such contributions will leverage the development of novel VR applications as they will allow optimizing multisensory experiences and, consequently, achieving more credible VEs.

\section{Related Work}

No studies were found that studied presence regarding such a diversity of multisensory stimuli combined in credible environments. We aim to explore the combination of vision, audition, vibration, passive haptic feedback, wind, and scent. Multisensory environments provide more sensory information to the user than the typical combination of visual and auditory stimuli. The addition of olfactory or haptic stimuli in conjunction with visual and audio stimuli should result in a more immersive, coherent, and credible experience, thus possibly raising the level of presence experienced even more.

As for smell, studies have revealed that scents can be used to enhance the sense of presence [20], [28]. Scents can also be used to induce multiple emotions in the user and thereby change his behaviour accordingly. For instance, the scent of burning, chemicals or other odors associated with dangerous situations can change users' behaviours [29]. Haptic feedback such as wind and heat is often used in virtual environments, resulting in an increase in the sense of presence [20], [30], [31], [32], [33].

Another example of a haptic stimulus is the use of real objects as passive haptics which we particularly study in this work. Meehan et al. [34] and Insko et al. [35] placed a $3.8 \mathrm{~cm}$-high ground board on the floor where the participant could walk on it. In a virtual pit room, the participant would not only see the pit but feel the ledge with their feet. Differences were found between the passive haptics and nonpassive haptics conditions for heart rate, skin conductance and behavioural presence (behaviours associated with presence), indicating a higher presence when passive haptics were present. The reported presence, although $p>0.05$, showed a strong trend in the same direction. Differently to our experimental design, the use of passive haptics in this study was for participants to feel the ledge and not climb up and down from the ground board like in our VR game.

A study conducted by Dinh et al. [20] showed that the more sensory stimuli are added, the greater the sense of presence is. In their work, participants reported a greater sense of presence when presented with auditory and tactile stimuli in addition to visuals. However, these visuals were not sufficiently advanced compared to today's reality. The recent technology advances allow for a much better simulation of reality, which can change how subjects interpret and react to multisensory stimuli. Moreover, there was a major limitation regarding the locomotion, which could have induced cybersickness (which we explain in further detail later in this section). Users were unable to control their location and could only use head movements. We overcome this limitation in our work by allowing players to physically walk throughout the game and be fully in control of their motion. Dinh et al. also studied olfactory cues (scent of coffee), which showed non-significant results, yet they tended to raise the sense of presence when introduced. However, the way the scent was delivered was intrusive: "via a small oxygen mask connected to a canister of coffee grounds and a small pump". In our work, we tried to deliver the stimuli in the least intrusive way possible to avoid breaking the subject's immersion. Perhaps if the scent of coffee in Dinh et al.'s work had been delivered in a more natural and less intrusive way, there might have been significant differences in presence. The authors further concluded that sensory stimuli work in a simple additive way regarding presence.

However, a more recent study from Fröhlich and Wachsmuth [21] indicates that increasing the quantity of stimuli does not necessarily result in higher presence. They studied the impact of the combination of modalities in virtual worlds on the sense of presence. The combination of visual and auditory stimuli, or vision with haptics (wind and tactile feedback vibration at the tip of the fingers), did not seem to impact the level of presence over visual stimuli alone. Nevertheless, the combination of all stimuli resulted in a significantly higher level of presence. Furthermore, their experiment was conducted in the Cave Automatic Virtual Environment (CAVE) system. The authors justify that these results are probably because users were expecting more when presented with just one additional stimulus. When all the stimuli were delivered, users' expectations were better met and, thus, a significant difference in presence arose.

There were limitations to the experiment regarding the variety of stimuli delivered and, again, locomotion. That study does not comprise as many stimuli as ours, and because a CAVE system was used, walking-in-place locomotion was adopted [36]. This method, although better than virtual flying, is inferior to real walking [37] in its simplicity and naturality. To address the number of stimuli delivered in our work, we merged the different stimuli presented in the works by Dihn et al. and by Fröhlich and Wachsmuth and also added passive haptics.

Regarding the coherence of the stimuli, Bessa et al. [17] conducted an experiment to verify how non-coherent stimuli could impact the sense of presence. The results showed differences between conditions, where the condition that represented lower credibility had lower presence scores. The results further indicated that in the more credible scenario, participants performed more reflexive motor actions that consisted of involuntary muscle responses to a particular stimulus. The authors concluded that perceived credibility is positively correlated with the sense of presence.

Work has been conducted demonstrating that users can behave in the virtual environment as they would in real life. For instance, in the study by Fröhlich and Wachsmuth [21], participants were presented a pit with a glass floor allowing 
them to step on it, but almost no one did so. Another example is the study conducted by Mel Slater et al. [38], which was inspired by the Stanley Milgram obedience study. In the original study, participants would appear to give electric shocks to a stranger when he would give incorrect answers to a set of questions on the behest of an authority figure. The results of the replicated experiment in a virtual environment indicate that even though participants knew the experience was not real, they showed signs of growing anxiety when giving shocks.

One important possible drawback when using VR applications is cybersickness symptoms. Users may start to feel nausea, headaches, ocular discomfort, and disorientation, among other symptoms, which can influence the performance, presence, and overall experience of the VR users.

It is important to keep track of cybersickness throughout the experiments [18], [39], [40], so we use it as a control variable. Cybersickness can be evaluated through questionnaires such as the Simulator Sickness Questionnaire (SSQ) [41]. These symptoms usually occur when there is a conflict between the visual and vestibular systems [42], [43], [44], [45]. Here, the type of locomotion strongly influences cybersickness. Studies show that real walking [37], [46], where the user physically walks, is the best type of locomotion. It induces lower cybersickness scores than others types of locomotion and is the most natural and easy way of moving in a VE, avoiding possible serious sensory conflict.

Regarding the impact of cybersickness on presence, Witmer and Singer [47] and Busscher, de Vliegher, Ling, \& Brinkman, [48] concluded in their studies that individuals who report more cybersickness symptoms will report less presence. However, Lin et al. [49] found a positive correlation between cybersickness and presence scores.

As far as the state of the art, we can find works that study multisensory stimuli in VR. However, none has combined such a variety of different stimuli, simultaneously delivered, in one credible VR environment. Thus, we conducted an experimental study to address this gap and investigate whether the addition of multisensory stimuli of wind, vibration, passive feedback, and scent causes an increase in presence in an already-credible environment. We also study the effect of passive haptics on presence in credible environments. In the state of the art there is work [34], [35] that has already investigated passive haptics and presence. However, it consists in feeling the edge of a pit with one's feet and ours consists in climbing up and down from boards placed on the ground. Also, the credibility of our environment is much higher. Cybersickness was also included in our study as a control variable to verify if the delivery of our multisensory stimuli could introduce cybersickness symptoms, which could compromise the results.

\section{Methods and Materials}

As described below, an experimental cross-sectional study, of comparative character and between-group design, was conducted to investigate the impact of multisensory stimuli on the sense of presence. Cybersickness was included in this study as a control variable, as it helps to understand if multisensory stimuli were congruent.

\subsection{Sample}

We used a convenience sample comprising 37 participants (27 men and 10 women). The participants were randomly divided into 3 groups (Table 1$)$ : The first group $(\mathrm{N}=12)$ was the control group, in which only the visual and auditory stimuli were used. The second group $(\mathrm{N}=13)$ performed experiments with passive haptics in addition to vision and audio. The passive haptics consisted of a wooden board placed on the ground. The third group $(\mathrm{N}=12)$ performed with all multisensory stimuli (visual, auditory, passive haptic, vibration, wind, and scent).

The participant's age ranged from 17 to 44 years old $(\mathrm{M}=22.350 ; \mathrm{SD}=4.529)$. Most of the participants were university students. Regarding past usage of VR, 24 participants ( $64.9 \%$ of the sample) had tried VR before. To better characterize our sample, the following data was collected from a sociodemographic questionnaire. Regarding participants' education, $51.4 \%$ (19) of participants had completed high school, and the remaining 48.6\% (18) had completed higher education. In regard to their experience with computers, participants described their knowledge on a 5-point scale (None, Basic, Intermediate, Good, Excellent): 3 participants $(8.1 \%)$ rated their experience with computers as being basic, 5 participants $(13.5 \%)$ as being intermediate, 19 participants $(51.4 \%)$ as being good and 10 participants (27\%) as being excellent. Their level of knowledge about virtual reality was also measured on a 5-point scale (None, Basic, Intermediate, Good, Excellent): 1 participant (2.7\%) rated their level of knowledge about VR as none, 16 participants $(43.2 \%)$ as being basic, 12 participants (32.4\%) as being intermediate, 7 participants (18.9\%) as being good and 1 participant $(2.7 \%)$ as being excellent.

\subsection{Instruments}

The virtual stimulus used as a case study was a VR game designed by the research team. This VR game was developed using Unity ${ }^{\circledR} 2017$ and was entitled "Illusions". The development of our game was inspired by a pilot study that was conducted with five different VR games to identify which features of game mechanics and environment should be incorporated into our virtual stimuli. The five games were available at the online platform Steam [50] and they were the following: "The Abbots Book", "Budget Cuts", "The price of freedom", "Quanero", and "Endless Night". These games offered insight about the scary, adventure, detective and action game genres in VR. From this pilot, the games that felt more immersive were the ones with a dark and scary environment. Also, the game mechanics and narrative ideas from all games were taken into consideration and the most suitable for our game were adopted.

As a result, "Illusions" presents a dark environment with moments of suspense and multiple challenges to be

TABLE 1

Grouping of the participants per experimental scenario.

\begin{tabular}{llll}
\hline & Control Scenario & Passive Haptics & Multisensory \\
\hline$N$ & 12 & 13 & 12 \\
\hline
\end{tabular}



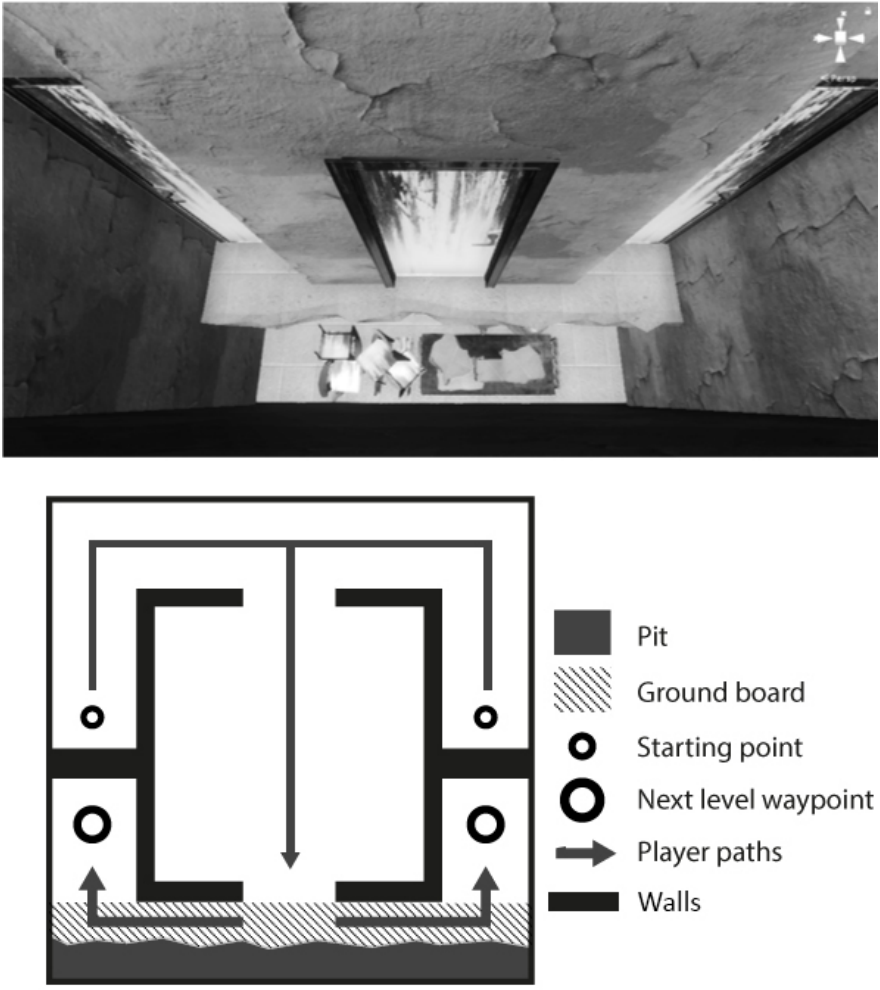

Fig. 1. Example of one of the game's level layouts, where the goal is to reach one of the next level waypoints. TOP: View of the pit room in the second level as configured in the control scenario. The player leaves through the middle door (which corresponds to the opening at the bottom of the floorplan in the bottom image) and has to choose between going left or right. BOTTOM: Simplified blueprint of the same level.

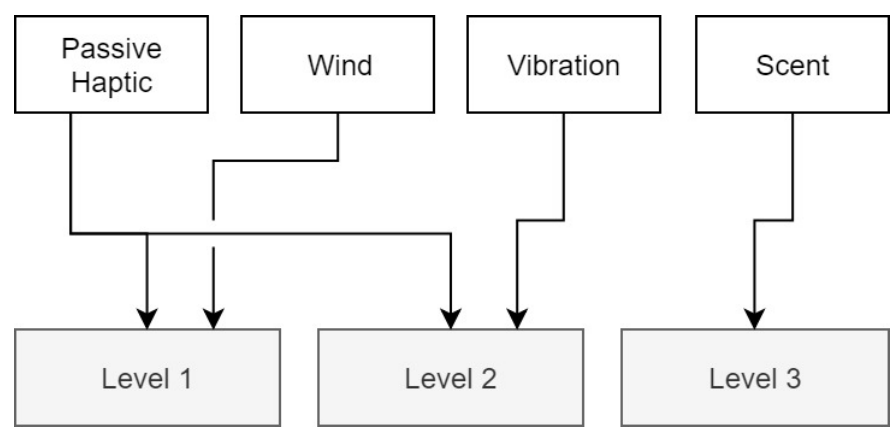

Fig. 2. Distribution of the stimuli through the different levels for the MS condition. For the PH condition, only the passive haptic stimulus is presented whereas in the CS condition none of the identified stimuli is presented.

overcome by the player. Due to the dark environment in several moments of the game, the participants can make use of a flashlight to see in the dark. The audio depicts a scary sound ambience and sounds generated by events (doors and drawers opening, objects colliding and others). The sound was spatialized, and a low pass filter and volume attenuation were applied to sounds that were occluded by doors and walls. The real name and age of the participant were also included in the game in a "Patient Chart" as a part of the narrative. The action of Illusions happens inside an abandoned hospital and features three levels. In each level, there will be a locked door that requires the player to find the key. Participants were able to interact with several objects (opening doors by grabbing the knob and pulling, grabing objects and moving them in 6-DOF...). The difficulty increases each level, and more thought is needed to discover where the key is hidden. Each level has two possible routes that the player can choose to advance to the next level (Fig. 1). Both paths will lead to the same destination (next level) but through opposite sides. If a player chooses to go left, they will start the next level in the left starting point (viceversa). Different stimuli were delivered according to the experimental scenario in each level. In the passive haptic scenario, a wooden board was positioned on the ground in both levels one and two. In the multisensory scenario, the first level had the passive haptic stimulus and a haptic stimulus wind coming from one window in the hallway. In the second level, there was again a passive haptic stimulus, but here the users would feel vibrations when stepping on top of it. The third level had a burning smell coming from one side of the hallway. The distribution of the stimuli can be better visualized in Fig. 2. Regarding avatar representation, the participants could only see their hands in the VE, represented by the HTC Vive controllers.

The levels were designed in a way that allows the player to physically walk through the entire game in an area of 4 $\mathrm{m} \times 4 \mathrm{~m}$. Physical walking movement has been proven by the state of the art to be the best locomotion type.

As for the collection of the data, we adopted the translated and validated Portuguese version of the IPQ [51], the IPQp [52], to assess presence and its subscales. This questionnaire incorporates 14 items using a 5-point Likert scale with the following subscales: Spatial Presence (the feeling of being physically present in the virtual environment), Experienced Realism (the subjective experience of realism), Involvement (the attention that is given to the environment and the level of involvement experienced) and Presence (the global sense of presence). Cybersickness was evaluated using the SSQ questionnaire [41]. The questionnaire was translated into the Portuguese language and is composed of 16 items corresponding to a set of symptoms. These can be classified as None, Slight, Moderate, Severe. This questionnaire included the following subscales: nausea (nausea, salivation, burping, stomach awareness), oculomotor discomfort (eye strain, difficulty focusing, blurry vision), disorientation (vertigo and dizziness) and cybersickness (global cybersickness).

\subsection{Equipment}

The virtual experience was operated using a desktop computer with the following specifications: CPU Intel ${ }^{\circledR}$ Core $^{\mathrm{TM}}$ i75820K @ 3.30 GHz, 32 GB RAM, Geforce ${ }^{\circledR}$ GTX 1080Ti. The frame rate was stable at $90 \mathrm{fps}$, and there was no lack of computing performance which could compromise the experiment. The visual stimulus and interaction with the experience were delivered through participants' use of the HTC Vive system [53]. For audio, headphones with active noise cancelling were used. The passive haptic stimulus was delivered by a wooden board placed on the ground at a 10$\mathrm{cm}$ elevation to serve as a step. We mounted a transducer [54] in the same ground board to deliver vibration when the user was above the board (Fig. 3). The release of wind 


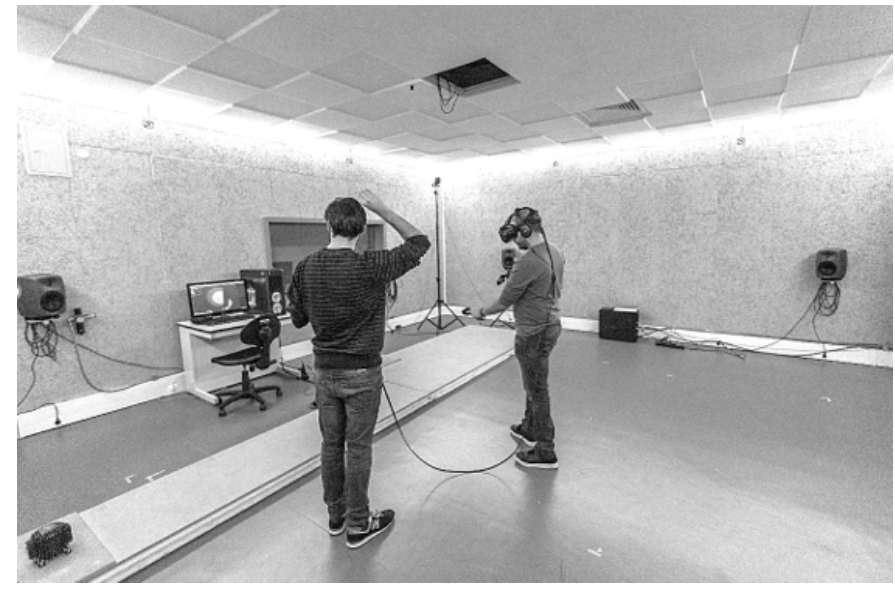

Fig. 3. Experimental apparatus (illustrative photo).

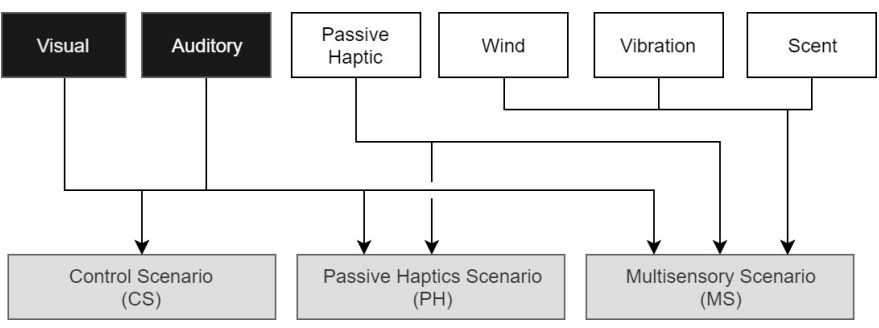

Fig. 4. Distribution of the stimuli through the different conditions.

was achieved by compressed air through a hose. Regarding the olfactory stimulus, the machine SensoryCo SmX-4D [55] was used to yield the smell of burning. In conjunction with the pressurized air, the scent was oriented directly to the player.

\subsection{Variables}

In this study, we considered one independent variable (Multisensory Stimulation) with three levels: Control scenario, Passive Haptics scenario, and Multisensory scenario (Fig.4). All three scenarios are described as above:

- CS: Control scenario $(\mathrm{n}=12)$;

- Scenario where participants performed the experience with only visual and auditory stimuli.

- PH: Passive Haptics scenario ( $n=13)$,

- In this scenario, in addition to visual and auditory stimuli, we added a wooden board placed on the ground to serve as a passive haptic stimulus (Fig. 3).

- MS: Multisensory scenario ( $\mathrm{n}=12)$.

- Scenario that gathers all the multisensory stimuli of the experience (visual, auditory, passive haptic, vibration, wind, and burning scent).

The two dependent variables considered were:

- Presence and its subscales (Spatial Presence, Experienced Realism, Involvement) measured using the IPQp;

- Cybersickness and its subscales (Nausea, Oculomotor discomfort, Disorientation) measured using the SSQ;

\subsection{Procedure}

The experiences were conducted in a laboratory environment where the research team had full control over the ambient variables such as sound, temperature and light. Before each experience, participants were briefed and asked to complete a consent form and a sociodemographic questionnaire.

Due to the experimental apparatus, namely, the fact that a ground board to simulate a step was used in the $\mathrm{PH}$ and MS conditions, we informed participants to be careful as some virtual elements could, in fact, exist in reality and to interact with them accordingly (e.g., physically climb steps).

In the $\mathrm{PH}$ and MS scenarios, the ground board position was synced with the virtual environment in such a way participants could see the board in the same place that it was placed in the experimental room. This procedure ensured that when participants were climbing the virtual step, they were also climbing the real step safely (Fig. 3).

As the MS scenario considers smell, participants were asked if they had limitations in breathing through the nose or detecting smells so to not compromise the impact of the olfactory stimuli.

All participants began the virtual experience facing the same orientation and in the centre of the virtual room (that matches with the centre of the experimental room). Without their knowledge, their name and age were introduced at the beginning of the virtual experience into the application so the correct data could appear in the Patient Chart that was shown in the middle of the virtual experience. Note that the name was not registered after the experience because the participants were only identified by an ID number to ensure their anonymity. This procedure was conducted regardless of the experiment condition. The subjects did not remove the VR equipment until the end of the game. Throughout the whole experience, the participants were always accompanied by a researcher to provide physical support if needed. The cables were managed in such a way that they would not interfere in the participant's experience. There was no time limit: the experience ended when the player managed to reach the end of the game. The average time participants took to finish the game was approximately 14 minutes, with the slowest taking 31 minutes and the fastest 9 minutes to complete.

After finishing the virtual experience, participants were asked to complete the IPQp, SSQ. Lastly, a debriefing was conducted to collect data about the experience (assess if participants understood the story, if they felt present, and any further details that they could share).

\subsection{Data Analysis}

A preliminary analysis of the data was conducted to verify its normal distribution $(\mid$ Skewness $\mid<3$ and $\mid$ Kurtosis $\mid<$ 10) [56]. From the analysis, it was verified that there were no outliers in the sample $(\mathrm{N}=37)$. Thus, the results were analysed using parametric statistics [57], namely, multivariate analysis of variance. Partial eta-squared $\left(\eta_{p}^{2}\right)$ was reported as a measure of effect size between groups according to the following rule of thumb: small $(>0.01)$, medium (> $0.06)$ and large $(>0.14)$. To determine statistically significant differences, a confidence interval of $95 \%(p<0.05)$ was considered. All of these statistical analyses were conducted using the SPSS software. 


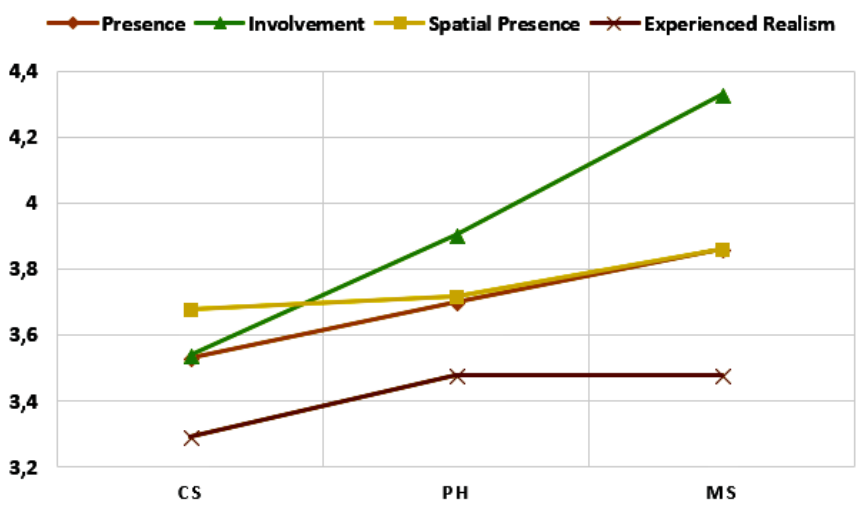

Fig. 5. Presence subscales means between conditions with a significant difference in Involvement between CS and MS.

\section{Results}

\subsection{Differences across conditions regarding the Sense of Presence}

To compare the impact of multisensory stimuli across the different experimental scenarios, a MANOVA analysis was conducted for Presence and the corresponding subscales from IPQp. The results showed no differences for $p<$ $0.05\left(F(8,62)=1.206 ; p=0.310 ; W_{i l k}^{\prime} s \Lambda=0.749 ; \eta_{p}^{2}=\right.$ $0.135 ; O . P .=0.509)$. Due to a moderate effect and observed power, we conducted a univariate analysis (ANOVA) (Table 2 ). The test showed statistically significant differences for Involvement $\left(F(2,34)=4.509 ; p=0.018 ; \eta_{p}^{2}=0.210 ; O . P .=\right.$ $0.732)$. The results for Presence $(F(2,34)=2.134 ; p=$ $\left.0.134 \eta_{p}^{2}=0.112 ; O . P .=0.407\right)$ presented a moderate effect, so further post hoc analysis was conducted.

Post hoc tests using the Bonferroni correction were performed to identify in which experimental scenarios the differences occurred. The results revealed that these differences exist between the conditions CS and MS $(p=0.015)$ (Table 3). The MS (multisensory scenario) had a higher score on the Involvement subscale than the CS (control scenario). The results are summarized in Fig.5.

\subsection{Differences across conditions regarding cybersick- ness}

To investigate the existence of differences between conditions regarding cybersickness and its subscales, a MANOVA was performed. No differences were found for $p<0.05$ $\left(F(6,64)=1.097 ; p=0.374 ;\right.$ Wilk's $s=0.822 ; \eta_{p}^{2}=$ $0.093 ;$ O.P. $=0.402$ ). Further analysis was performed through ANOVAs (Table 4). The results seem to indicate statistically significant differences for cybersickness with a large associated effect $(F(2,34)=3.366 ; p=$ $\left.0.046 ; \eta_{p}^{2}=0.165 ; O P=0.596\right)$. The subscale Nausea $\left(F(2,34)=1.929 ; p=0.161, \eta_{p}^{2}=0.102, O . P .=0.372\right)$ presents no differences, although there is a moderate effect. The subscales Oculomotor Discomfort $(F(2,34)=$ $\left.3.210 ; p=0.053 ; \eta_{p}^{2}=0.159 ; O . P .=0.574\right)$ and Disorientation $\left(F(2,34)=2.952 ; p=0.660, \eta_{p}^{2}=0.148 ; O . P .=0.537\right)$ show no differences, but both present a large effect.

Due to the large and moderate effects verified, post hoc tests were performed using the Bonferroni correction to confirm where the differences occur. The results revealed no statistically significant differences (for $p<0.05$ ) for any of the subscales in any of the experimental scenarios. Such suggests a type I error (false positive).

\section{Discussion}

\subsection{Presence}

In this work, we studied the effect of credible multisensory stimuli on the sense of presence in a credible VE. In H1 we speculated that coherent multisensory stimuli in credible environments would increase the sense of presence. We verified statistically significant differences between the control scenario and the multisensory scenario in presence (for $p<0.05$ ) in the subscale Involvement. By observing the means, we verified that the multisensory scenario achieved the highest levels of presence, followed by the passive haptics scenario and the control scenario. We can see the score increase graphically in Fig. 5 and in percentages in Table 5 between pairs of conditions. The results partially confirm the H1. There was indeed an increase in presence, but it was only significant in the Involvement subscale. These results are partially in line with the study by Dinh et al. [20] that indicates that the more stimuli are added, the higher the sense of presence reported. Fröhlich and Wachsmuth [21] also studied the combination of multisensory stimuli in the sense of presence and obtained similar results: only when combining all the stimuli was the reported sense of presence higher. However, in our work, using a credible VE, the Involvement subscale was the only dimension of presence that had significant differences. Such a result could suggest that it is more difficult to raise presence scores significantly by adding multisensory stimuli in credible environments than in non-credible environments. There could be various reasons for this result. One reason could be that users are already very immersed in the VE and that the delivery of a simple multisensory stimulus (such the passive haptic stimulus in this study) could pass unnoticed or does not have sufficient impact to raise users' sense of presence. It was only in the condition that gathered all the multisensory stimuli that users reported a significant change in presence, and it was only in the Involvement scale.

Involvement measures the attention devoted to the $\mathrm{VE}$ and the involvement experienced. In other words, higher involvement scores indicate that users are more captivated by the virtual environment and less aware of the real world. There is the possibility that a passive haptic stimulus such as the step aroused a sense of alertness in the participants. After the first encounter with a virtual object that is also physically present, users asked themselves what else might truly be present. Thus, the level of alertness may increase, and subjects may begin to pay more attention to what else could truly be present physically and, consequently, attain higher involvement scores. We need further studies to measure alertness to investigate whether or not these justifications are valid. Another reason for a possible increase of involvement score may be simply due to the higher number of stimuli in the experience. As players experienced an increasing number of different stimuli throughout the game, they may have begun to be more attentive to what else is present. Another reason may lie in the fact that 
TABLE 2

Univariate analysis for the independent variable Multisensory Stimulation regarding Presence and its subscales across all three conditions. Each line is the result of the ANOVA in each Presence subscale. Means and standard deviations also included.

\begin{tabular}{|c|c|c|c|c|c|c|c|c|c|}
\hline & \multicolumn{2}{|c|}{ Control Scenario } & \multicolumn{2}{|c|}{ Passive Haptics } & \multicolumn{2}{|c|}{ Multisensory } & $p$ & $\eta_{p}^{2}$ & O.P. \\
\hline Presence & 3.530 & 0.486 & 3.703 & 0.278 & 3.863 & 0.403 & 0.134 & 0.112 & 0.407 \\
\hline Spatial Presence & 3.681 & 0.134 & 3.718 & 0.155 & 3.861 & 0.536 & 0.568 & 0.033 & 0.137 \\
\hline Experienced Realism & 3.292 & 0.852 & 3.481 & 0.563 & 3.479 & 0.815 & 0.776 & 0.015 & 0.087 \\
\hline
\end{tabular}

TABLE 3

Bonferroni Post-hoc analysis for the independent variable Multisensory Stimulation regarding Presence and Involvement subscales.

\begin{tabular}{llll}
\hline & CS vs. PH & CS vs. MS & PH vs. MS \\
\hline Presence & $p=0.842$ & $p=0.140$ & $p=0.959$ \\
Involvement & $p=0.512$ & $p=0.015$ & $p=0.319$ \\
\hline
\end{tabular}

the wide range of multisensory stimuli overrode the realworld stimuli, causing users to not be aware of the real space they were in, instead focusing their attention on the VR application. However, the conditions of the laboratory where we conducted the experiences were well controlled. Stimuli such as temperature, sound and light were under full control of the research team and stayed the same during the experiences.

We suspected in $\mathrm{H} 2$ that by adding passive haptics alone, we could verify a significant increase in presence. This was due to the better and more realistic locomotion which allows users to interact more naturally with the environment and better feel the passive haptics. However, similar to Meehan et al. [34] and Insko et al. [35], the use of passive haptics did not provoke significant differences in the reported presence.

Although there was a positive trend, we could not confirm H2. These results lead us to believe that passive haptics by itself may not be a differentiating stimulus, but, as the literature suggests, it could be used additively to other stimuli to increase presence.

Overall, we can observe a positive trend in the presence subscales from the control scenario to passive haptics and from passive haptics to the multisensory scenario, although without statistical significance. We noticed that in the subscale Experienced Realism, the means between the PH and MS conditions were almost identical. This dimension of presence translates to how real the virtual world was for the subjects-in other words, how consistent the virtual experience was with a real-world experience. There is a small increase in this subscale only from the CS to the $\mathrm{PH}$ condition. A possible explanation is the possibility of climbing up and down from the ground board, which is an action consistent with a real-world situation. Because between the PH and MS conditions the ground board was always present, the means remained very close, which leaves us to question why the other stimuli did not impact this subscale. We theorize that this lack of impact was because the absence of wind, vibration, and scent was equally coherent with their presence in the VE. For example, the first level included wind in the MS condition by having a window open. Participants could see the window, but this does not mean that they necessarily expected wind to come through it. It is credible and coherent to see a window open and either to feel or not to feel wind passing through it. In this line of thought, an example of a situation where the presence of wind could impact the experienced realism is if we had a curtain that appeared to be moving due to the passage of wind. Therefore, we suspect that the observed level of experienced realism remained the same between $\mathrm{PH}$ and MS because the VE is experienced as real whether or not these stimuli are present in our VE. Additionally, we suspect that we did not see significant changes in this subscale from CS to PH because the impact of the passive haptic stimulus itself was not strong enough to cause significant changes. Perhaps if we also had a real desk that corresponded to a virtual desk, or even a real swivel chair that the user could also see virtually, sit in or move, it could have significantly improved the experienced realism. A possible cause for not being verified a statistically significant increase in presence just through the addition of passive haptics could be due to the participant's inability to see their virtual feet. When on flat ground, people wearing HMD do not usually watch their feet, but when negotiating a step up or down, they may be prompted to look at their feet and notice that they cannot be seen. Such could be considered a conflicting cue against reality, resulting in a decrease in the realism of the scenario, offsetting the increased realism of the step co-existing in the real and virtual environments. Also, the ground board that was providing the passive haptic stimulus did not correspond entirely to the visible shape of the ledge, thus possibly adding a sense of unreality. Further studies are needed to confirm this.

We must bear in mind that the various subscales of presence are correlated with one another. Thus, our arguments explaining the lack of significant differences, or the reasons behind the means to a certain subscale, can also apply in part to the rest of the subscales. Moreover, there are no statistically significant differences beyond the Involvement subscale in presence across conditions, and this is simply an analysis of the observed trends. We discuss them because they can show new lines of research through which to further investigate the discussed possibilities.

\subsection{User Behaviour}

Regarding the observed behaviour when in the pit room (level 2), the participants acted similarly to how we would expect them to act in reality. We systematically saw participants from all conditions trying to lean against the virtual wall so they would not fall into the pit, even though there was actually no wall. This behaviour shows that participants 
TABLE 4

Univariate analysis for the independent variable Multisensory Stimulation regarding cybersickness and its subscales across all three conditions. Each line is the result of the ANOVA in each cybersickness subscale. Means and standard deviations also included.

\begin{tabular}{|c|c|c|c|c|c|c|c|c|c|}
\hline & \multicolumn{2}{|c|}{ Control Scenario } & \multicolumn{2}{|c|}{ Passive Haptics } & \multicolumn{5}{|c|}{ Multisensory } \\
\hline & Mean & S.D & Mean & S.D & Mean & S.D & $p$ & $\eta_{p}^{2}$ & O.P. \\
\hline Cybersickness & 19.323 & 19.192 & 7.480 & 7.785 & 7.792 & 8.656 & $0.046^{*}$ & 0.165 & 0.596 \\
\hline Nausea & 14.310 & 22.466 & 5.137 & 9.229 & 3.975 & 4.912 & 0.161 & 0.102 & 0.372 \\
\hline Oculomotor discomfort & 15.160 & 14.088 & 5.831 & 7.025 & 6.317 & 8.449 & 0.053 & 0.159 & 0.574 \\
\hline Disorientation & 23.200 & 18.133 & 9.637 & 11.900 & 11.600 & 14.336 & 0.066 & 0.148 & 0.537 \\
\hline
\end{tabular}

* Post-hoc tests revealed no statistically significant differences between scenarios. This suggests a type I error (false positive).

TABLE 5

Difference of the scores in percentage between conditions of all presence subscales.

\begin{tabular}{llll}
\hline & CS-PH & PH-MS & CS-MS \\
\hline Presence & $5 \%$ & $4 \%$ & $9 \%$ \\
Spatial Presence & $1 \%$ & $4 \%$ & $5 \%$ \\
Involvement & $10 \%$ & $11 \%$ & $22 \%$ \\
Experienced Realism & $6 \%$ & $0 \%$ & $6 \%$ \\
\hline
\end{tabular}

felt levels of presence high enough to cause them to act realistically. That display corroborates the results found by Fröhlich and Wachsmuth [21] where participants in a pit room acted like the pit was real and avoided stepping on it, even though they knew there was no pit in reality. Whether there were multisensory stimuli or not, this situation evoked a realistic response from the subjects.

Similar results to ours can be found in work by Meehan et al. [34] and Insko et al. [35]. They concluded that participant's behavioural presence was increased when presented with a ground board where they could feel the ledge with their feet. However, in our study, the participants could not feel the ledge with their feet in the game's second level because the board itself was large and covered the virtual pit they were seeing. They could only feel the board when stepping up and down from it. In our study, participants did not see their feet in the VE, only their hands which were represented by the HTC Vive controllers. We observed that participants were hesitant when stepping in and out of the ground board and in the ledge part of the game. We suspect that the inexistence of virtual feet could have caused extra effort from users to understand where they were putting their feet. However, in the rest of the game, in flat surfaces, they seemed to have no problems walking, even without virtual feet.

\subsection{Cybersickness}

In our game, we tried to make all the multisensory stimuli coherent with one another and with what the player could actually feel in an analogous real-world situation. By using real-walking and congruent multisensory stimuli, we aimed to mitigate possible cybersickness symptoms by avoiding sensory conflict. Despite our best efforts in such, we considered cybersickness as a control variable to ensure that it would not influence the levels of presence. If cybersickness symptoms were to significantly arise between conditions, it could influence presence scores, thus compromising the results. The study of the control variable cybersickness did not reveal statistically significant differences between any condition, and it showed very low mean scores as well. This suggests that cybersickness did not influence presence scores significantly. Similar results regarding the nonexistence of significant differences between CS and PH can also be found in Meehan et al. [34] and Insko et al. [35].

Although not significant, results suggest that there is a difference between CS and the rest of the scenarios. This could be due to the use of passive haptics since, when adding passive haptics (PH scenario), cybersickness scores lowered, and remained identical when we added more stimuli (MS). Nevertheless, further studies are needed to verify if this is true and, in case it is, to identify the causes for such.

\section{CONCLUSION}

This study's main objective was to investigate whether multisensory stimuli could raise the sense of presence in a credible VE, and if passive haptics alone could be a differentiating stimulus. The results showed that only the combination of all multisensory stimuli increased the involvement of the users, thus leading to more attention devoted to the VR environment. Such a finding could suggest that it is more difficult to raise presence through multisensory stimulation in credible environments than in non-credible ones. A passive haptics stimulus did not significantly increase presence by itself, but a positive trend was found when this stimulus was added. Cybersickness was also studied to verify that our method of delivering the multisensory stimuli did not compromise the experience in this regard. Our multisensory stimuli did not have an impact on cybersickness across conditions.

The importance of such study has led the research team to plan future work, such as the relevance of each stimulus in the sense of presence: if the stimuli are relevant for the user's task, one hypothesis is that these stimuli can significantly benefit the sense of presence. Another challenge will be to extend our studies with an additional stimulus such as temperature, as it can add an extra layer of coherence and credibility, leading to higher levels of presence. In all cases, we will ensure that every participant feels all the stimuli that he or she is intended to.

Several questions were raised in the discussion of the results that require further studies to verify. Future work must be conducted to investigate if the various multisensory stimuli have any impact on subjects' alertness. We must investigate if the absence of multisensory stimuli implying lower realism of the VE (e.g., seeing a window curtain moving but feeling no wind) can have an impact on the 
experienced realism and overall presence. It should also be investigated if the addition of passive haptics resulted in lower cybersickness scores (although not significant in this experiment). Future research directions shall also study the addition of a virtual body or virtual feet to verify its effects on the passive haptics potential to increase presence.

There were limitations with the ground board, as subjects were not able to feel the ledge in front of them with their feet. Thus, when testing the existence of that ledge with their feet, they would feel the flat surface they were already on. In further studies, the ground board should be cut in a way that resembles the ledge subjects see in the VE. The experimental design did not allow us to remove the passive haptics stimulus from the multisensory condition to investigate whether or not the significance still holds. This would help us understand how passive haptics impacts presence when used with other stimuli. This was due to the fact that we used the ground board to deliver vibration. If we eliminated the passive haptics from MS scenario, we also could not deliver vibration.

\section{ACKNOWLEDGEMENTS}

This work is financed by the ERDF - European Regional Development Fund through the Operational Programme for Competitiveness and Internationalisation - COMPETE 2020 Programme and by National Funds through the Portuguese funding agency, FCT - Fundação para a Ciência e a Tecnologia within project POCI-01-0145-FEDER-028618 entitled PERFECT - Perceptual Equivalence in virtual Reality For authEntiC Training.

\section{REFERENCES}

[1] I. Messinis, D. Saltaouras, P. Pintelas, and T. Mikropoulos, "Investigation of the Relation Between Interaction and Sense of Presence in Educational Virtual Environments," in 2010 International Conference on e-Education, e-Business, e-Management and e-Learning. IEEE, 2010, pp. 428-431.

[2] T. Monahan, G. McArdle, and M. Bertolotto, "Virtual reality for collaborative e-learning," Computers \& Education, vol. 50, no. 4, pp. 1339-1353, May 2008.

[3] L. Freina and M. Ott, "A literature review on immersive virtual reality in education: state of the art and perspectives," in The International Scientific Conference eLearning and Software for Education, vol. 1, 2015, p. 133.

[4] L. Barbosa, P. Monteiro, M. Pinto, H. Coelho, M. Melo, and M. Bessa, "Multisensory virtual environment for firefighter training simulation: Study of the impact of haptic feedback on task execution," in $201724^{\circ}$ Encontro Português de Computação Gráfica e Interação (EPCGI). IEEE, oct 2017, pp. 1-7.

[5] T. P. Grantcharov, V. B. Kristiansen, J. Bendix, L. Bardram, J. Rosenberg, and P. Funch-Jensen, "Randomized clinical trial of virtual reality simulation for laparoscopic skills training," British Journal of Surgery, vol. 91, no. 2, pp. 146-150, jan 2004.

[6] F. Aïm, G. Lonjon, D. Hannouche, and R. Nizard, "Effectiveness of Virtual Reality Training in Orthopaedic Surgery," Arthroscopy: The Journal of Arthroscopic \& Related Surgery, vol. 32, no. 1, pp. 224-232, jan 2016.

[7] A. David Cheok, M. Haller, O. Noel Newton Fernando, and J. Prasad Wijesena, "Mixed Reality Entertainment and Art," International Journal of Virtual Reality (IJVR), vol. 08, no. 2, pp. 83-90, Dec. 2015.

[8] M. Zyda, "From visual simulation to virtual reality to games," Computer, vol. 38, no. 9, pp. 25-32, Sep. 2005.

[9] P. Fuchs, G. Moreau, and P. Guitton, Virtual Reality: Concepts and Technologies, 1st ed. CRC Press, Jul. 2011.
[10] M. Slater and S. Wilbur, "A Framework for Immersive Virtual Environments (FIVE): Speculations on the Role of Presence in Virtual Environments," Presence: Teleoperators and Virtual Environments, vol. 6, no. 6, pp. 603-616, Dec. 1997.

[11] M. Slater, "A Note on Presence Terminology," Presence Connect, vol. 3, 2003.

[12] P. Bouvier, "The five pillars of presence: guidelines to reach presence," SPAGNOLLI, A. et GAMBERINI, L., éditeurs: Proceedings of Presence, pp. 246-249, 2008.

[13] W. Ijsselsteijn and G. Riva, "Being there : concepts, effects and measurements of user presence in synthetic environments," in Emerging communication: studies in new technologies and practices in communication, G. Riva, F. Davide, and W. A. IJsselsteijn, Eds. IOS Press, Jan. 2003, vol. 5, pp. 3-16.

[14] M. Lombard and T. Ditton, "At the Heart of It All: The Concept of Presence," Journal of Computer-Mediated Communication, vol. 3 , no. 2, pp. 0-0, Jun. 2006.

[15] R. Skarbez, F. P. Brooks Jr, and M. C. Whitton, "A survey of presence and related concepts," ACM Computing Surveys (CSUR), vol. 50, no. 6, p. 96, 2018.

[16] M. Slater and M. Usoh, "Presence in immersive virtual environments," in Proceedings of IEEE Virtual Reality Annual International Symposium, Sep 1993, pp. 90-96.

[17] M. Bessa, M. Melo, A. A. de Sousa, and J. Vasconcelos-Raposo, "The effects of body position on Reflexive Motor Acts and the sense of presence in virtual environments," Computers \& Graphics, vol. 71, pp. 35-41, Apr. 2018.

[18] M. Melo, J. Vasconcelos-Raposo, and M. Bessa, "Presence and cybersickness in immersive content: Effects of content type, exposure time and gender," Computers \& Graphics, vol. 71, pp. 159-165, Apr. 2018.

[19] T. B. Sheridan, "Musings on Telepresence and Virtual Presence," Presence: Teleoperators and Virtual Environments, vol. 1, no. 1, pp. 120-126, jan 1992.

[20] H. Q. Dinh, N. Walker, L. F. Hodges, C. Song, and A. Kobayashi, "Evaluating the importance of multi-sensory input on memory and the sense of presence in virtual environments," in Proceedings IEEE Virtual Reality (Cat. No. 99CB36316). IEEE Comput. Soc, 1999, pp. 222-228.

[21] J. Fröhlich and I. Wachsmuth, "The Visual, the Auditory and the Haptic - A User Study on Combining Modalities in Virtual Worlds," in Virtual Augmented and Mixed Reality. Designing and Developing Augmented and Virtual Environments. Springer Berlin Heidelberg, 2013, pp. 159-168.

[22] M. Feng, A. Dey, and R. W. Lindeman, "An initial exploration of a multi-sensory design space: Tactile support for walking in immersive virtual environments," in 2016 IEEE Symposium on 3D User Interfaces (3DUI). IEEE, Mar. 2016, pp. 95-104.

[23] P. G. de Barros and R. W. Lindeman, "Performance effects of multi-sensory displays in virtual teleoperation environments," in Proceedings of the 1st symposium on Spatial user interaction - SUI '13. ACM Press, 2013.

[24] "Definition of CREDIBILITY." [Online]. Available: https://www. merriam-webster.com/dictionary/credibility

[25] M. Slater and M. Usoh, "Body Centred Interaction in Immersive Virtual Environments," in Artificial Life and Virtual Reality, vol. 1. John Wiley and Sons, 1994, pp. 125-148.

[26] M. Slater, "Place illusion and plausibility can lead to realistic behaviour in immersive virtual environments," Philosophical Transactions of the Royal Society B: Biological Sciences, vol. 364, no. 1535, pp. 3549-3557, Dec. 2009, 22.

[27] N. Magnenat-Thalmann, H. Kim, A. Egges, and S. Garchery, “Believability and Interaction in Virtual Worlds," in 11th International Multimedia Modelling Conference. IEEE, 2005, pp. 2-9.

[28] W. Barfield and E. Danas, "Comments on the Use of Olfactory Displays for Virtual Environments," Presence: Teleoperators and Virtual Environments, vol. 5, no. 1, pp. 109-121, Jan. 1996.

[29] G. Ghinea and O. A. Ademoye, "Olfaction-enhanced multimedia: perspectives and challenges," Multimedia Tools and Applications, vol. 55, no. 3, pp. 601-626, Dec. 2011, 33.

[30] R. J. Stone, "Haptic feedback: a brief history from telepresence to virtual reality," in Haptic Human-Computer Interaction, G. Goos, J. Hartmanis, J. van Leeuwen, S. Brewster, and R. Murray-Smith, Eds. Berlin, Heidelberg: Springer Berlin Heidelberg, 2001, vol. 2058, pp. 1-16, 27. 
[31] C. Cellini, L. Kaim, and K. Drewing, "Visual and Haptic Integration in the Estimation of Softness of Deformable Objects," $i$ Perception, vol. 4, no. 8, pp. 516-531, Dec. 2013, 28.

[32] M. A. Srinivasan and C. Basdogan, "Haptics in virtual environments: Taxonomy, research status, and challenges," Computers $\mathcal{E}$ Graphics, vol. 21, no. 4, pp. 393-404, Jul. 1997, 25.

[33] E.-L. Sallnäs, K. Rassmus-Gröhn, and C. Sjöström, "Supporting presence in collaborative environments by haptic force feedback," ACM Transactions on Computer-Human Interaction, vol. 7, no. 4, pp. 461-476, Dec. 2000.

[34] M. Meehan, B. Insko, M. Whitton, and F. P. Brooks, Jr., "Physiological measures of presence in stressful virtual environments," vol. 21, no. 3. New York, NY, USA: ACM, Jul. 2002, pp. 645-652.

[35] B. E. Insko, M. Meehan, M. Whitton, and F. Brooks, "Passive haptics significantly enhances virtual environments," PhD Thesis, University of North Carolina at Chapel Hill, 2001.

[36] M. Slater, M. Usoh, and A. Steed, "Taking steps: The influence of a walking technique on presence in virtual reality," $A C M$ Transactions on Computer-Human Interaction (TOCHI), vol. 2, pp. 201-219, 121995

[37] M. Usoh, K. Arthur, M. C. Whitton, R. Bastos, A. Steed, M. Slater, and F. P. Brooks, Jr., "Walking > walking-in-place> flying, in virtual environments," in Proceedings of the 26th Annual Conference on Computer Graphics and Interactive Techniques, ser. SIGGRAPH '99. New York, NY, USA: ACM Press/AddisonWesley Publishing Co., 1999, pp. 359-364. [Online]. Available: http://dx.doi.org/10.1145/311535.311589

[38] M. Slater, A. Antley, A. Davison, D. Swapp, C. Guger, C. Barker, N. Pistrang, and M. V. Sanchez-Vives, "A Virtual Reprise of the Stanley Milgram Obedience Experiments," PLOS ONE, vol. 1, no. 1 p. e39, Dec. 2006, 23.

[39] D. Narciso, M. Bessa, M. Melo, A. Coelho, and J. VasconcelosRaposo, "Immersive $360^{\circ}$ video user experience: impact of different variables in the sense of presence and cybersickness," Universal Access in the Information Society, Oct. 2017.

[40] S. A. A. Naqvi, N. Badruddin, A. S. Malik, W. Hazabbah, and B. Abdullah, "Does 3d produce more symptoms of visually induced motion sickness?" in Engineering in Medicine and Biology Society $(E M B C), 2013$ 35th annual international conference of the IEEE. IEEE, 2013, pp. 6405-6408.

[41] R. S. Kennedy, N. E. Lane, K. S. Berbaum, and M. G. Lilienthal, "Simulator Sickness Questionnaire: An Enhanced Method for Quantifying Simulator Sickness," The International Journal of Aviation Psychology, vol. 3, no. 3, pp. 203-220, Jul. 1993, 40.

[42] S. Davis, K. Nesbitt, and E. Nalivaiko, "A systematic review of cybersickness," in Proceedings of the 2014 Conference on Interactive Entertainment, ser. IE2014. New York, NY, USA: ACM, 2014, pp. 8:1-8:9.

[43] J. J. LaViola, "A discussion of cybersickness in virtual environments," ACM SIGCHI Bulletin, vol. 32, no. 1, pp. 47-56, Jan. 2000, 1.

[44] S. Sharples, S. Cobb, A. Moody, and J. R. Wilson, "Virtual reality induced symptoms and effects (VRISE): Comparison of head mounted display (HMD), desktop and projection display systems," Displays, vol. 29, no. 2, pp. 58-69, Mar. 2008, 31.

[45] J. Reason and J. Brand, Motion Sickness. Academic Press, 1975.

[46] P. Monteiro, D. Carvalho, M. Melo, F. Branco, and M. Bessa, "Application of the steering law to virtual reality walking navigation interfaces," Computers \& Graphics, vol. 77, 102018.

[47] B. G. Witmer and M. J. Singer, "Measuring Presence in Virtual Environments: A Presence Questionnaire," Presence: Teleoperators and Virtual Environments, vol. 7, no. 3, pp. 225-240, Jun. 1998, 21.

[48] B. Busscher, D. de Vliegher, Y. Ling, and W.-P. Brinkman, "Physiological measures and self-report to evaluate neutral virtual reality worlds," Journal of CyberTherapy and Rehabilitation, vol. 4, no. 1, pp. $15-25,2011$

[49] J. J. W. Lin, H. B. L. Duh, D. E. Parker, H. Abi-Rached, and T. A. Furness, "Effects of field of view on presence, enjoyment, memory, and simulator sickness in a virtual environment," in Proceedings IEEE Virtual Reality 2002, 2002, pp. 164-171, 4.

[50] [Online]. Available: https://store.steampowered.com/about/

[52] J. Vasconcelos-Raposo, M. Bessa, M. Melo, L. Barbosa, R. Rodrigues, C. M. Teixeira, L. Cabral, and A. A. Sousa, "Adaptation and validation of the igroup presence questionnaire (ipq) in a por-
[51] T. Schubert, F. Friedmann, and H. Regenbrecht, "The experience of presence: Factor analytic insights," Presence: Teleoperators and Virtual Environments, vol. 10, no. 3, pp. 266-281, Jun. 2001, 44. tuguese sample," Presence: Teleoperators and Virtual Environments, vol. 25, no. 3, pp. 191-203, 2016.

[53] "VIVETM | Discover Virtual Reality Beyond Imagination," 2017. [Online]. Available: https://www.vive.com/us/

[54] "TheButtKicker.com," 2017. [Online]. Available: https: //thebuttkicker.com/

[55] "SmX-4d Series," 2018. [Online]. Available: https://www. sensorycots.com/smx-4d-series

[56] J. Marôco, Análise de equações estruturais: Fundamentos teóricos, software E aplicações. ReportNumber, Lda, 2010.

[57] G. M. Sullivan and A. R. Artino Jr, "Analyzing and interpreting data from likert-type scales," Journal of graduate medical education, vol. 5, no. 4, pp. 541-542, 2013.

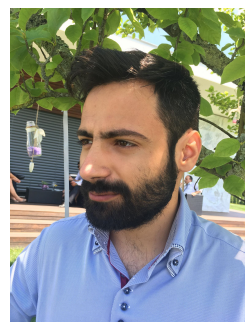

Guilherme Gonçalves is a MSc in Multimedia by the University of Trás-os-Montes e Alto Douro (UTAD), Vila Real, Portugal, where he acquired his bachelor's degree in 2015. His research in terests are mainly Multisensory Virtual Reality.

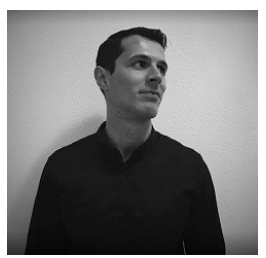

Miguel Melo is a Post-doc researcher at INESC TEC, specialized in Computer Graphics. He is manager of the Multisensory Virtual Reality Laboratory and his research interests are Computer Graphics, HDR, and Multisensory Virtual Reality.

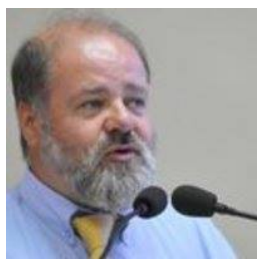

José Vasconcelos-Raposo is a full-professor in the Department of Education and Psychology at UTAD, specialized in Performance Psychology. $\mathrm{He}$ is a member of INESC TEC -UTAD and his research interests are in the use of technology in improving human performance applied in the most diverse fields of applications such as sports, health care, or training and certification.

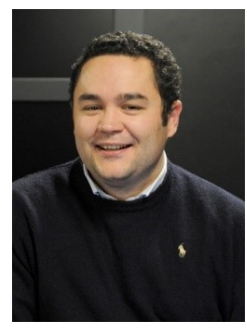

Maximino Bessa is Assistant Professor, with Habilitation, of the Department of Engineering of the University of Trás-os-Montes and Alto Douro, Portugal, Senior Researcher of INESC TEC since 2009 and Director of the Multisensory Virtual Reality Laboratory MASSIVE. He's Member of the Eurographics Association since 2003 and Vice-President of the Portuguese Computer Graphics Chapter for the period 2016-2018. 